\title{
Assessment of Simple Models for Molecular Simulation of Ethylene Carbonate and Propylene Carbonate as Solvents for Electrolyte Solutions
}

\author{
Mangesh I. Chaudhari ${ }^{1}$ Ajay Muralidharan ${ }^{2}$ Lawrence R. Pratt ${ }^{2}$. \\ Susan B. Rempe ${ }^{1}$
}

Received: 25 July 2017 / Accepted: 23 January 2018 / Published online: 12 February 2018 (C) The Author(s) 2018. This article is an open access publication

\begin{abstract}
Progress in understanding liquid ethylene carbonate (EC) and propylene carbonate (PC) on the basis of molecular simulation, emphasizing simple models of interatomic forces, is reviewed. Results on the bulk liquids are examined from the perspective of anticipated applications to materials for electrical energy storage devices. Preliminary results on electrochemical double-layer capacitors based on carbon nanotube forests and on model solid-electrolyte interphase (SEI) layers of lithium ion batteries are considered as examples. The basic results discussed suggest that an empirically parameterized, non-polarizable force field can reproduce experimental structural, thermodynamic, and dielectric properties of EC and PC liquids with acceptable accuracy. More sophisticated force fields might include molecular polarizability and Buckingham-model description of inter-atomic overlap repulsions as extensions to Lennard-Jones models of van der Waals interactions. Simple approaches should be similarly successful also for applications to organic molecular ions in EC/PC solutions, but the important case of $\mathrm{Li}^{+}$deserves special attention because of the particularly strong interactions of that small ion with neighboring solvent molecules. To treat the $\mathrm{Li}^{+}$ions in liquid EC/PC solutions, we identify interaction models defined by empirically scaled partial charges for ion-solvent interactions. The empirical adjustments use more basic inputs, electronic structure calculations and ab initio molecular dynamics simulations, and also experimental results on
\end{abstract}

This article is part of the Topical Collection "Modeling Electrochemical Energy Storage at the Atomic Scale"; edited by "Martin Korth".

Susan B. Rempe

slrempe@sandia.gov

1 Center for Biological and Engineering Sciences, Sandia National Laboratories, Albuquerque NM 87185, USA

2 Department of Chemical and Biomolecular Engineering, Tulane University, New Orleans LA 70118, USA 
$\mathrm{Li}^{+}$thermodynamics and transport in EC/PC solutions. Application of such models to the mechanism of $\mathrm{Li}^{+}$transport in glassy SEI models emphasizes the advantage of long time-scale molecular dynamics studies of these non-equilibrium materials.

Keywords Li-ion battery $\cdot$ Molecular dynamics simulations · Propylene carbonate $\cdot$ Ethylene carbonate

\section{Introduction}

An electrochemical voltage window is a primary concern for electrical energy storage applications of an electrolyte system, e.g., for lithium ion batteries (LIBs) and electrochemical double-layer capacitors (EDLCs). That voltage window is a primary issue for the energy density, but also a consideration in addressing safety. As a practical matter, that voltage window concern excludes aqueous electrolyte solutions [1]. Non-aqueous electrolyte solutions [2] are well-recognized, but the molecular simulation experience with those systems is orders of magnitude more limited than for aqueous systems [3-5]. This is partly due to the broad importance of water as a liquid medium [6], but also due to vast chemical and compositional variety relevant for non-aqueous systems [7-10].

Understanding that daunting range of chemical possibilities, including assessment of voltage windows, has put natural emphasis on screening enabled by electronic structure computations of theoretical chemistry [11-13]. But macroscopic characteristics of these liquids - such as phase diagrams, dielectric responses, and fluid phase kinetics - are relevant too, and direct numerical simulation of the solutions help in that screening. Careful molecular simulation often requires validation of models and techniques, consideration of a range of thermodynamic states, and understanding the scale limitations of the results. Therefore it can be helpful for simulation work to examine relevant cases in depth to complement screening approaches.

Recent work has aimed at filling in the simulation basis for study of non-aqueous electrolyte solutions, i.e., for ethylene carbonate (EC) and propylene carbonate (PC) systems, at a molecular level (Fig. 1). This report collects and discusses recent

Fig. 1 Chemical structures of ethylene carbonate (EC) and propylene carbonate $(\mathrm{PC})$
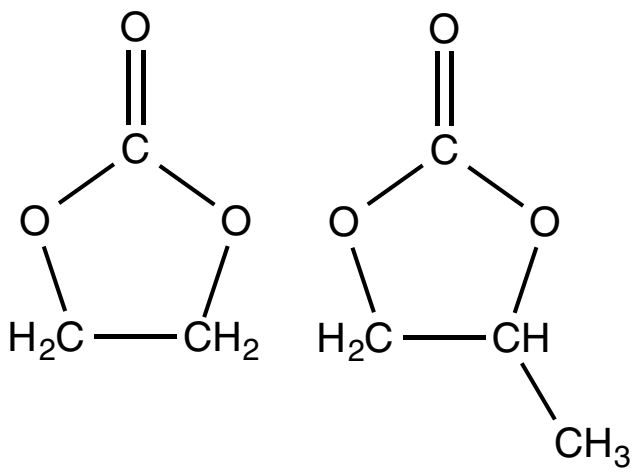
simulation results on these solvents to identify basic research that might help in further design of materials.

Molecular simulation is a useful tool for development of new materials. Development of effective, simplified molecular simulation models would enable enlightening simulation of dynamical phenomena of specific interest for electrical energy storage systems, i.e., transport through inhomogeneous or non-equilibrium materials, or of annealing processes involving those non-equilibrium materials.

Electrochemical double-layer capacitors (EDLCs), or supercapacitors [14], present cases of inhomogeneous materials. EDLCs based on carbon nanotube (CNT) forests provide a specific setting for molecular-scale examination of the dynamics of propylene carbonate solutions of complex salts [15], a setting where the underlying microstructure is comparatively unambiguous [16]. We note that a variety of solvent/electrolyte systems, including ionic liquids [10], are commonly considered with EDLCs. Nevertheless, molecular-scale descriptions that might explain the dependence on electrode microscale structures or of the rates of charging/discharging have not been carried through. Synthesis of CNTs [17-19] with well-characterized molecular-scale microstructure should assist in establishing the molecular theories sought to understand these systems fully.

An even more prominent example of important molecular-scale kinetics involving inhomogeneous or non-equilibrium materials is that of $\mathrm{Li}^{+}$ion transport through the solid electrolyte interphase (SEI) of lithium ion battery (LIB) [20]. The SEI of an LIB forms during initial charging/discharging cycles [21-23]. Solution components decompose [24], forming a passivating anode layer. $\mathrm{Li}^{+}$ions travel through that complex organic material. The composition of the SEI depends on a variety of factors, including solvent and additives, ions, anode material, voltage, temperature and the use history. Understanding the atomic-scale mechanism of transport of $\mathrm{Li}^{+}$ion through the SEI should assist in development of high-performance LIBs, through better characterization and control of the SEI layer. Molecular simulations might help to bridge the learning gap [8, 9, 25, 26].

Molecular calculations and simulations are typically a necessary prerequisite for basic molecular theories. Molecular calculations span a daunting range of algorithmic techniques, and a daunting range of space and time scales. For example, quantum calculations track electrons and can characterize decomposition of electrolytes at anode surfaces [8, 9, 22, 27, 28]. These methods include ab initio molecular dynamics (AIMD), which have the drawback of computational expense and the concomitant limitation to small systems and time scales [29-31]. On the other hand, if chemical changes such as chemical bond rearrangement are essential to the study, AIMD provides natural perspectives on those phenomena.

Classical molecular dynamics simulation with model molecular force fields'force field molecular dynamics' (FFMD) — inhabit a broader region of the simulation scale. Useful force field models can span a broad range of possibilities, from frankly ad hoc models, to models that are recognized as coarse-grained on a pragmatic basis, then including progressively more complicated models. Electron coordinates can be reintroduced into FFMD approaches by development of models that include molecular polarizabilities. Polarization of that type has been considered important for this problem [32]. The polarizable force field of Borodin et al. [7]. for 
the carbonate solvents and SEI layer models $[25,26]$, has been applied to $\mathrm{LiBF}_{4}$ in PC [34].

Highly specialized force fields and parameters are not readily available for common molecular simulation packages. Additionally, their complexity limits their use for study of transport behaviors for novel materials that interrogate molecularly long correlation times. This overview focuses on the pragmatic middle of FFMD simulations focused on non-polarizable force fields with empirical parameters.

\subsection{Methods and Force Fields}

The FFMD simulations discussed specifically here were carried out using the GROMACS simulation package [35]. Details of the calculations differ slightly between cases, as noted with those discussions below, but were always obtained with force fields of standard non-polarizable format. We note the success of empirical force fields for liquid water [3-5]. EC and PC liquid results here used all-atom optimized potentials for liquid simulation (OPLS-AA) force fields and parameters [36]. There were several distinct reasons for these choices, beginning with simplicity and accessibility of these molecular simulation basics. Another reason for the present simple choice of force field model is that we emphasize liquid phase thermal properties that are statistical challenges for molecular simulation. Thus, the ability to examine sufficiently long statistical series is an important consideration. Finally, we note the sufficiency of empirical potential structure refinement (EPSR) modeling for reproducing the neutron diffraction results with exactly the same forms [37]. Thus, the present simple force field models should be sufficient also for those important data.

We identify secondary specific differences among those FFMD calculations discussed below, but we here provide several common features. These calculations adopted constant pressure simulation conditions with $p=1 \mathrm{~atm}$ on the basis of the Parrinello-Rahman barostat [38]. Temperatures were maintained with a Nosé-Hoover thermostat $[39,40]$. A time step of $1 \mathrm{fs}$ and time constant of $2.5 \mathrm{ps}$ were used for the thermostat and barostat, respectively. Periodic boundary conditions were applied standardly to simulate bulk liquid conditions. The particle mesh Ewald method was used to compute electrostatic interactions, and Lennard-Jones interactions were cutoff at $1.2 \mathrm{~nm}$. Long-ranged dispersion corrections were also applied. Bonds involving hydrogen atoms were constrained using the linear constraint solver (LINCS) algorithm [41].

Extensions of such a simple force field model are interesting for the chemical physics of these problems. A Buckingham [32,42] model of van der Waals repulsions is an extended feature that is likely to be generally helpful compared to a traditional $1 / r^{12}$ (Lennard-Jones) model. We comment further about that extension below when we discuss solvation of $\mathrm{Li}^{+}$ions in these carbonate liquids. Another interesting extension is the inclusion of solvent molecular polarizability in these force fields $[32,42]$. This feature is likely to be specifically important for electrolytesinvolving free ions-but we emphasize that dispersive van der Waals interactions are modeled separately in these forces fields. We note in passing that establishment 
of saturated solution conditions, perhaps involving ion pairing as a mechanism for phase separation, is primarily sensitive to (attractive) dispersive van der Waals interactions [43-45]. As with the common empirical force fields for liquid water simulation [3-5], the non-polarizable force field parameters should be recognized as effective values that approximate the outcomes obtained with more complicated force fields.

\subsection{Plan of this Report}

We will collect and discuss molecular simulation results on the EC and PC liquids and on solutions with electrolytes relevant to EDLC capacitors based on CNT forests [44, 46-48]. We will emphasize macroscopic characteristics that are often considered in discussions of such applications, particularly interfacial structure, dielectric responses, and molecular mobilities. In focusing here on the molecular basis of macroscopic characteristics of these liquids, the present report aims to complement the recent review [49] that emphasized synthesis and catalysis. We take up the example of EDLCs based on CNT forests where the liquid carbonate solutions are integral components. We then include $\mathrm{Li}^{+}$in these calculations $[32,50]$, leading to discussion of simulation of a model LIB SEI layer [42].

In closing this introduction, we reemphasize the common goal of devising highcapacity, fast-charging, safe electrical energy storage systems [21]. Commonly used electrical energy storage devices do present distinct material requirements. Therefore, breadth and fidelity in understanding possible materials should be an advantage. Indeed, other solvents have been considered in this context. For example, glycerol carbonate has been studied recently by neutron diffraction and modeling [37]. Nitriles have received extended study [1, 51-53], as has acetonitrile [54-62]. Of course, it is the non-aqueous conditions that are of interest here. But LIB applications have involved carbonate solvents, instead of nitriles [1], because of the role of carbonate molecules in chemical processes that form the SEI [63]. A recent study of PC/acetonitrile mixtures is striking due to the unusual solvent combination [64].

\section{Ethylene Carbonate and Propylene Carbonate Liquids}

The vapor pressures of EC and PC are low in regimes of practical use [44, 66], and thus they are strongly bound liquids. We further characterize [46] this 'strongly bound' quality by the ratio $T_{\mathrm{c}} / T_{\mathrm{t}}$ of their critical temperatures to their triplepoint temperatures. For the well-studied Lennard-Jones model liquid, this ratio is $T_{\mathrm{c}} / T_{\mathrm{t}}=1.9$. But for liquid $\mathrm{PC}$ and $\mathrm{EC}$, this ratio is $3.5(\mathrm{PC})$ and $2.3(\mathrm{EC})$. Acetonitrile and water, for which $T_{\mathrm{c}} / T_{\mathrm{t}} \approx 2.4$, provide further comparisons [46].

Estimation of $T_{\mathrm{c}}$ for our standard simulation model of PC, on the basis of extrapolation of liquid-vapor surface tensions (Fig. 2), is remarkably accurate. Study of those interfaces shows that the plane of the PC molecular is statistically oriented parallel to the interfacial plane, with the methyl group directed toward the vapor phase. Planar stacking persists when liquid PC contacts a planar graphite surface 

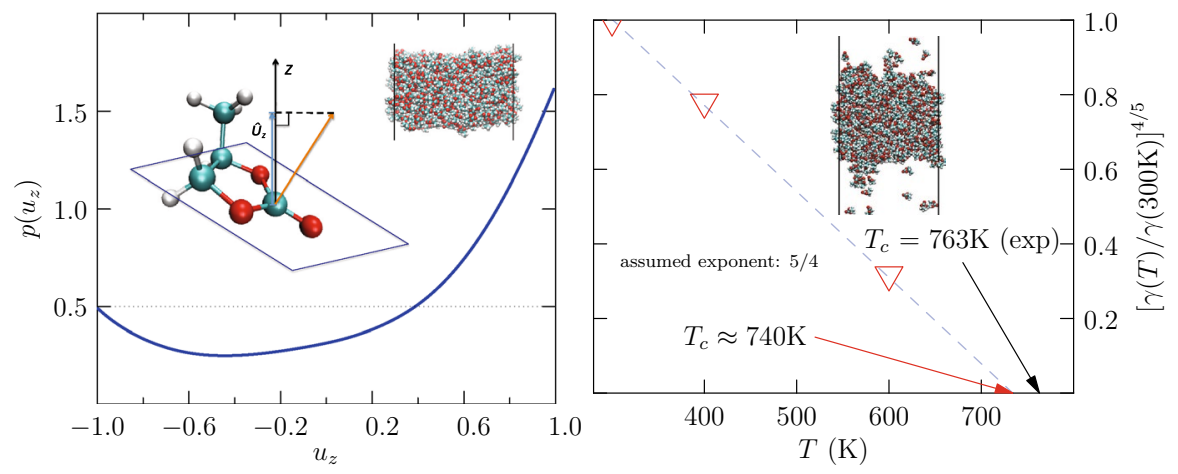

Fig. 2 (left) For PC molecules in the liquid-vapor interfacial layer at $T=300 \mathrm{~K}$, the probability density for projection of the unit vector normal to the carbonate plane onto the axis, perpendicular to the interface. The inset in upper-right corner of that panel indicates the slab geometry used for these calculations $[44,65]$. The 'outer' (vapor) direction corresponds to projections near 1.0. The most probable orientation aligns the carbonate plane parallel to the plane of the interface, with the methyl group extended toward the vapor phase. $u_{z}>0.5\left(\theta<60^{\circ}\right)$ for about $50 \%$ of interfacial PC molecules. (right) Liquid-vapor interfacial tensions for PC, extrapolated to estimate the critical temperature $T_{\mathrm{c}}$ as shown. The surface tension for the lowest $T$ shown here agrees well with the one experimental evaluation of that tension at $T=20^{\circ}$ C. The estimated vapor pressures for these cases are roughly correct [66]. The inset on the right panel is a configuration drawn from the $T=600 \mathrm{~K}$ calculation, which thus gives an indication of the co-existing vapor. These results together provide support for the observed interface structures and suggest that the balance of attractive intermolecular interactions is realistic

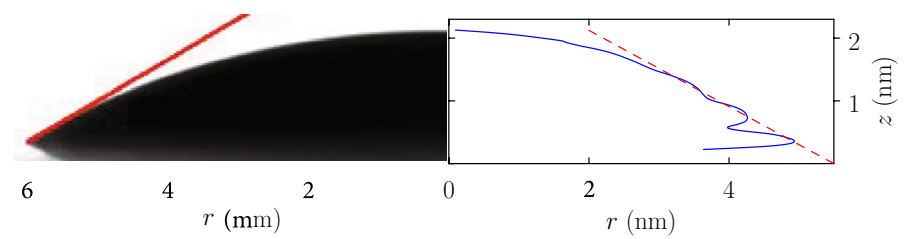

Fig. 3 PC droplet on graphite. The left side is the observed millimeter-scale droplet [44]. The blue curve on the right side is the nanometer-scale simulated droplet shape, obtained with adjustment of the van der Waals interaction to match the experimental contact angle as described in that reference. The fringe on the right side of the simulated droplet illustrates nanometer-scale molecular layering of PC molecules in contact with the graphite surface

(Fig. 3), except that the methyl group is preferentially oriented toward the liquid phase in that case [44]. The relative orientation of near-neighbor PC molecules in the liquid (Fig. 4) again display this rough planar stacking motif, with the two nearest neighbors corresponding to a plane above and a plane below a distinguished PC molecule. The dipole moments of these stacked neighbors tend to be anti-parallel and this has by now been experimentally confirmed on the basis of neutron diffraction from PC and glycerol carbonate [37].

The liquid density of standardly simulated PC at $p=1 \mathrm{~atm}$ is several percent denser [44] than the experimental value near $T=300 \mathrm{~K}$. The thermal expansion 


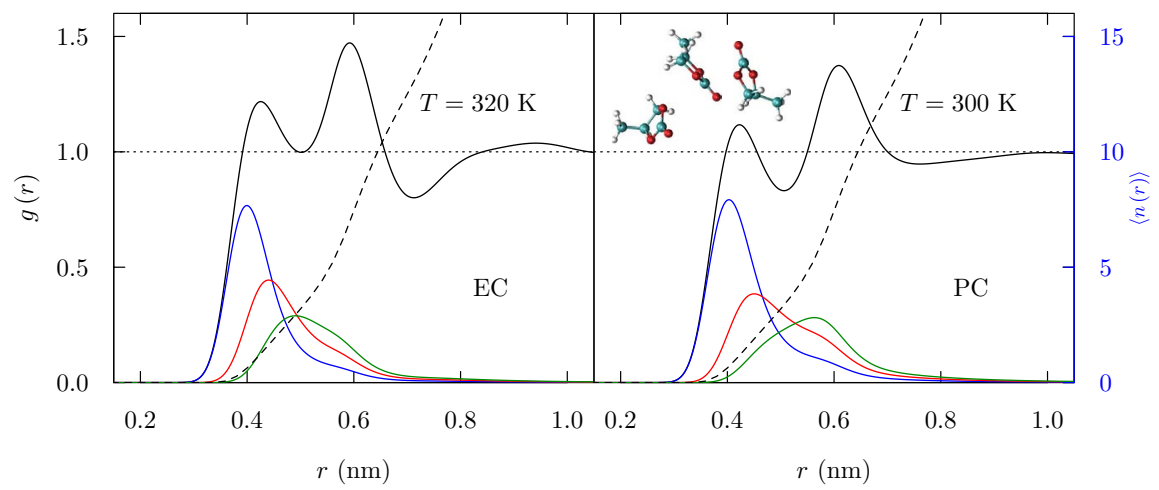

Fig. 4 Radial distribution functions associated with carbonyl carbon atoms. (black): Traditional radial distributions involving all carbonyl carbon atoms with the characteristic split primary peak, further quantified by the neighborship-ordered radial distribution functions for the closest (blue), 2nd-closest neighbors (red), and 3rd-closest neighbors (green) of a carbonyl carbon atom. The embedded molecular graphic illustrates that these close pairs exhibit stacking of carbonate planes and antiparallel dipole moments. The two closest neighbors saturate the closest peak of the traditional distribution function, and stacking of one plane on top and another on bottom achieves that

coefficient from the simulations under those conditions is highly accurate, although the isothermal compressibility is too small by about 50\% [44]. We expect that discrepancy in the isothermal compressibility would be improved by effective replacement of Lennard-Jones $1 / r^{12}$ repulsions by Buckingham repulsions [32, 42].

\subsection{Molecular Mobilities}

Here we characterize the mobilities of EC and PC molecules in their liquid by the slope of the mean-squared displacement (msd)

$$
\frac{\mathrm{d}\left\langle\Delta r(t)^{2}\right\rangle}{\mathrm{d} t} \sim 6 D
$$

at long times $t$, thus evaluating the self-diffusion coefficient, $D$. But we can take that characterization deeper (Fig. 5) before considering those mobilities broadly (Fig. 6). The step deeper is to consider the velocity autocorrelation function (acf) [67]

$$
C(t)=\langle\mathbf{v}(0) \cdot \mathbf{v}(t)\rangle /\left\langle v^{2}\right\rangle,
$$

from which the mobilities

$$
\frac{\mathrm{d}\left\langle\Delta r(t)^{2}\right\rangle}{\mathrm{d} t}=2\left\langle v^{2}\right\rangle \int_{0}^{t} C(\tau) \mathrm{d} \tau,
$$



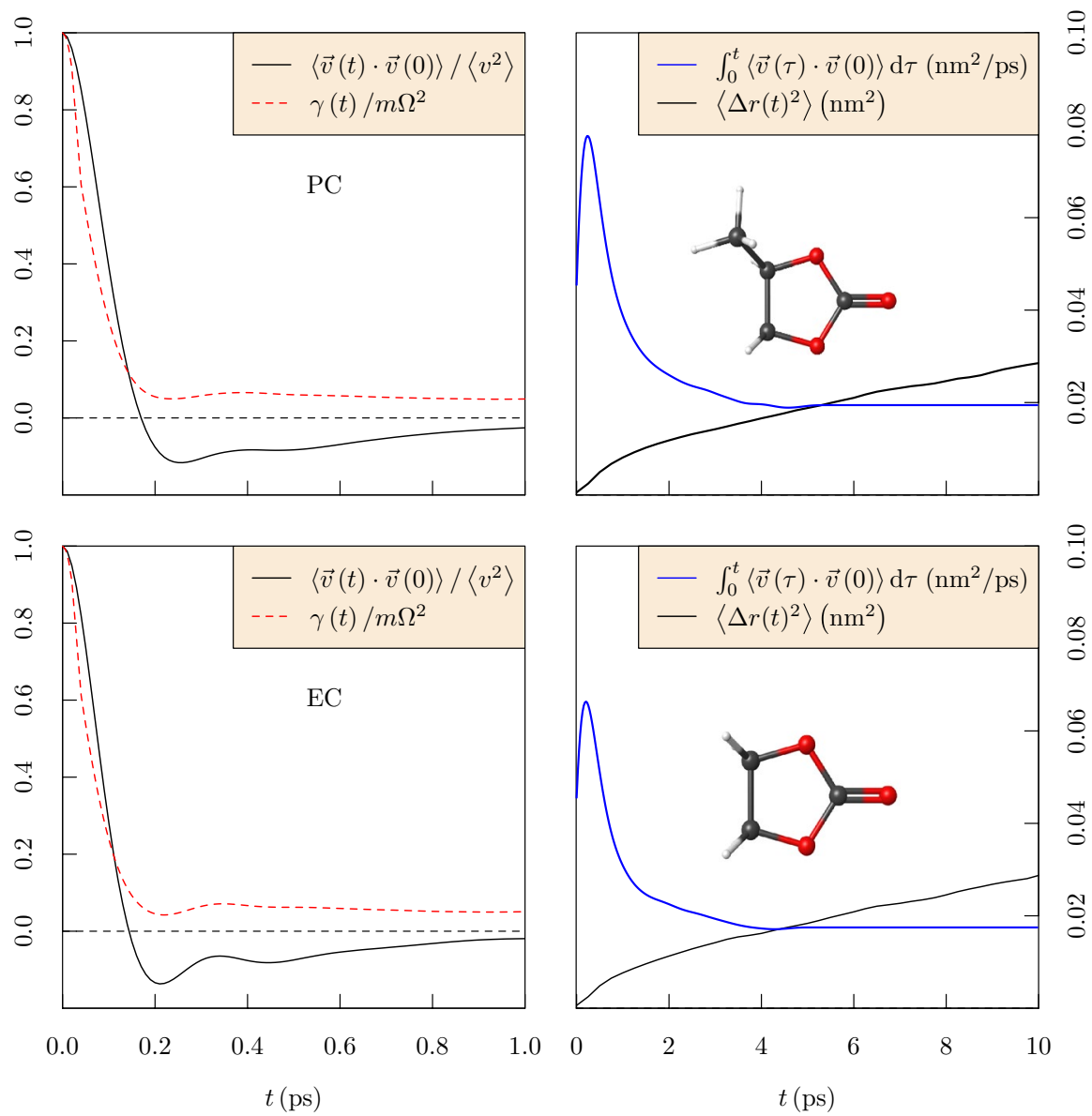

Fig. 5 (top-left) Time correlation functions associated with the velocity of the center of mass of PC molecules in liquid PC at $T=300 \mathrm{~K}$. For this strongly bound liquid, the velocity acf relaxes through negative values after several collision times. Consistent with this, the friction kernel $\gamma(t)$ relaxes over many collision times. This behavior has been attributed to attractive intermolecular interactions in these strongly bound liquids. (top-right) Mean-squared displacements of PC in liquid PC (dashed). The time derivative (following Eq. 3, blue solid curve) shows a prominent maximum due to the negative tail of the velocity autocorrelation function. After that maximum, the msd achieves a constant slope only slowly. This behavior is not evident, for example, in the hard-sphere liquid model [46]. (bottom) Similar plots for EC

may be then derived. Here the indicated velocities are those of the center of mass of the polyatomic EC/PC molecules. We also consider the friction kernel $\gamma(t)$ defined by [67]

$$
m \frac{\mathrm{d} C(t)}{\mathrm{d} t}=-\int_{0}^{t} \gamma(t-\tau) C(\tau) \mathrm{d} \tau
$$

with $m$ as the mass of the molecule. $\gamma(t)$ characterizes the random forces on these molecules, and 


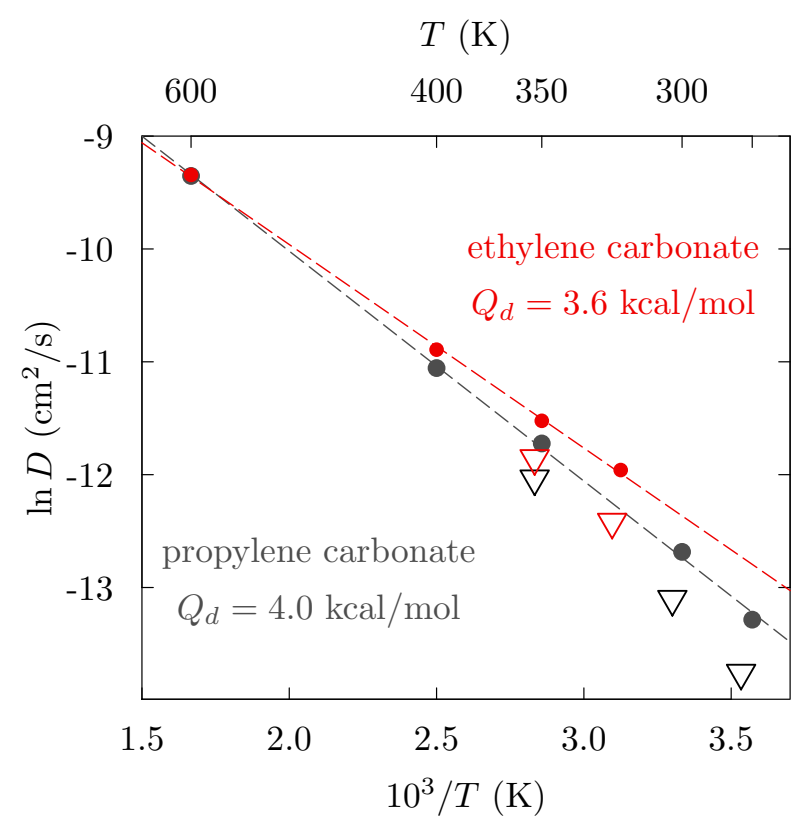

Fig. 6 Comparison of simulation [48] and experimental [70] values of EC/PC molecule self-diffusion coefficients, over a range of temperatures at constant ambient pressure. The experimental results were extracted from studies of $\mathrm{LiPF}_{6}$ solutions. $Q_{\mathrm{d}}$ is the activation energy parameter, $\ln D \propto-Q_{\mathrm{d}} / k_{\mathrm{B}} T$, identifying the slope of the indicated fitting lines

$$
\gamma(0)=m \Omega^{2}=\left\langle F^{2}\right\rangle / 3 k_{\mathrm{B}} T,
$$

emphasizes that connection with the forces on the molecules with $\Omega^{2}=\left\langle F^{2}\right\rangle / 3 m k_{\mathrm{B}} T$. An interesting observation for these strongly bound liquids [46, 47, 68] (Fig. 5) is that $C(t)$ exhibits a negative tail, i.e., relaxation through negative values for times longer than a collision time, and that negative tail substantially affects the evaluation of $D$ through Eq. (3). Contrary to the standard Langevin picture [67], the friction kernel $\gamma(t)$ also persists in relaxation over the same timescales of many collision times. That longer-timescale relaxation has been attributed to attractive intermolecular interactions in these strongly bound liquids [46, 47, 68, 69], particularly for the mobility of ions in solution for which long-ranged attractive forces are defining qualities. For the neutral PC molecule, indeed, that slowly relaxing tail of $\gamma(t)$ diminishes for the highest $T \mathrm{~s}$ considered [46, 47].

Experimental results for $D$ are only available for solutions of EC and PC with $\mathrm{LiPF}_{6}$ at $1 \mathrm{M}$ concentration [70]. Nevertheless, here we compare our computed results [48] to those mobilities (Fig. 6). Our results agree with those experimental values to within about a factor of 2 , satisfactory accuracy here. This encouraging comparison supports the use of the present non-polarizable force field in the studies reviewed below. The temperature dependence of $\ln D$ is linear in $1 / T$ over the range considered. 

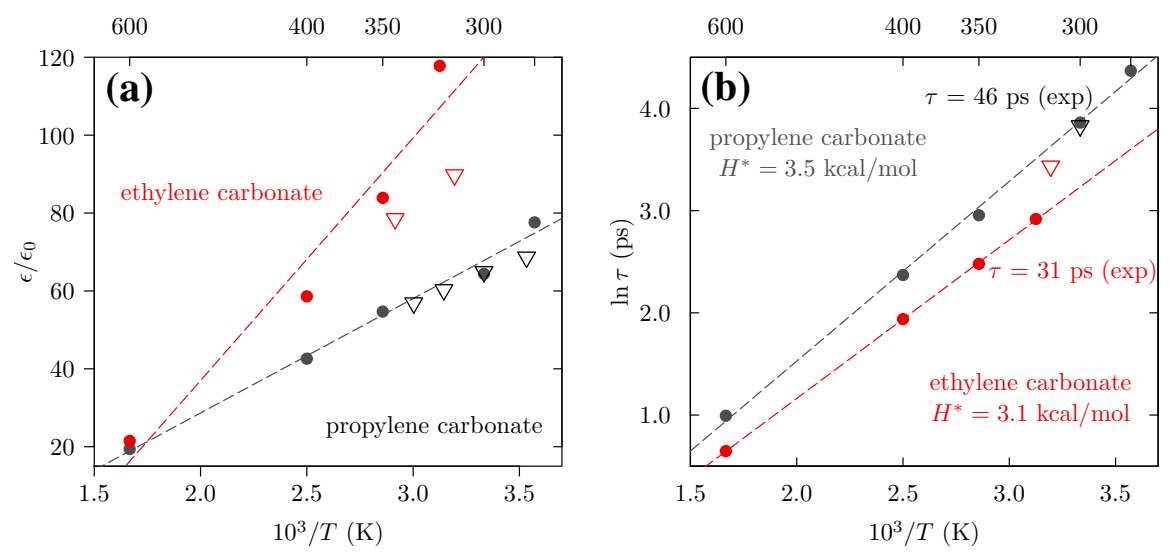

Fig. 7 a Static dielectric constants $\epsilon / \epsilon_{0}$ (in open triangles) and $\mathbf{b}$ relaxation times $\tau$ for EC (black) and PC $($ red $)$ at several temperatures. The experimental relaxation times indicated are those provided in the figure captions of the available experimental report [71], which incorporates an estimate of molecular polarizability and assumes a Debye relaxation model. Dashed lines are linear fits to the data, provided for visual guidance

\subsection{Dielectric Constants and Relaxation Times}

The high dielectric constants of EC and PC liquids correlate naturally with the solubility of strong electrolytes, including lithium salts, in these solvents [72]. Dielectric characteristics are thus properties of first interest for these liquids. Dielectric constants and relaxation times are strongly temperature-dependent, and that might have consequences for battery efficiency and safety. Here, the computed static dielectric constants (Fig. 7a) are in good agreement with the experimental values for both PC and EC [44, 48, 62, 65], though at the lowest temperature here, the discrepancy is nearly $30 \%$ (too large) for EC.

Dielectric relaxation characterizes the ability of the material polarization to follow a changing applied electric field [73]. Harmonic analysis of the field and the polarization leads to a frequency-dependent, complex dielectric constant [73-75]

$$
\epsilon(\omega)=\epsilon^{\prime}(\omega)-i \epsilon^{\prime \prime}(\omega) .
$$

with real and imaginary parts. $\epsilon^{\prime \prime}(\omega)$ describes frictional energy loss, and can be obtained from the polarization autocorrelation function [71, 75-77]

$$
P(t)=\langle M(0) M(t)\rangle /\left\langle M^{2}\right\rangle,
$$

of the total dipole moment at time $t, M(t)$, of the liquid. The acf $P(t)$ is then fit to the Fourier transform of a stretched exponential (or Kohlrausch-Williams-Watts, KWW) model [78],

$$
P_{\mathrm{KWW}}(t)=\exp \left[-(t / \tau)^{\beta}\right]
$$


where $\beta$ is the fitting parameter. Available experimental data and analysis of simulation data suggest that $\beta=1$ (Debye relaxation) is an accurate approximation for these systems.

The agreement between computed and experimental relaxation times is encouraging: $\tau=46$ ps from experiment on PC at room temperature compared to $\tau=48 \mathrm{ps}$ from these simulations. Note that extraction of the experiment relaxation times utilized models incorporating electronic polarizability $\epsilon(\omega=\infty) / \epsilon_{0}$, which we deliberately avoid here. Still, the relaxation times of 46 ps for PC, 31 ps for EC, and 8 ps for water emphasize [77] the comparative sluggishness of the carbonate solvents. This comparative sluggishness presents a severe challenge for simulation of these liquids on the basis of the more demanding simulation techniques such as AIMD.

The temperature dependence of the relaxation times

$$
\tau^{-1}=A \exp \left(-H^{*} / k_{\mathrm{B}} T\right),
$$

can be modeled with an activation energy, $H^{*}$. For simplicity, we assumed the preexponent factor $A$ to be independent of $T[71,79,80]$, and calculate $H^{*}$ from the slope of the Arrhenius plot,

$$
\ln \tau \propto H^{*} / k_{\mathrm{B}} T .
$$

The computed $H^{*}$ for EC $(3.1 \mathrm{kcal} / \mathrm{mol})$ and PC $(3.5 \mathrm{kcal} / \mathrm{mol})$ are within the range of activation enthalpies reported for liquid water $(2.8-4.5 \mathrm{kcal} / \mathrm{mol}$ for $278 \mathrm{~K}<T<348 \mathrm{~K}$ ) [81].

Though the present computational results cover a broad temperature range, fitting beyond a single activation energy has not been warranted so far. Still, it would be interesting, and maybe of practical relevance, for subsequent experiments and modeling to investigate super-cooled conditions more thoroughly.

\subsection{Non-linear Polarization Response}

The molecular electric fields at play on a molecular scale in ionic solutions are often much stronger than the laboratory electric fields used to measure dielectric constants. Thus, the equilibrium polarization responses to strong fields (Fig. 8) are often queried, even though statistical mechanical theories are less firmly grounded then. Indeed, the underlying theory of non-linear polarization response has been reexamined recently from a basic perspective [88-90]. Still, it is now clear that long-standing simple models [83] can do a good job of fitting non-linear polarization responses in controlled settings [62, 84, 91]. Interesting recent work [62] studied PC, EC, dimethyl carbonate (DMC), acetonitrile, and EC/DMC mixtures, and observed electrofreezing in several of these cases.

\subsection{Electrochemical Double-Layer Capacitor Based on CNT Forests}

Beyond clear potential for practical significance, EDLCs based on CNT forests offer the possibilities of better molecular-scale understanding of those solutions in contact with charged electrodes. This possibility is enabled by the simplicity of the electrode 


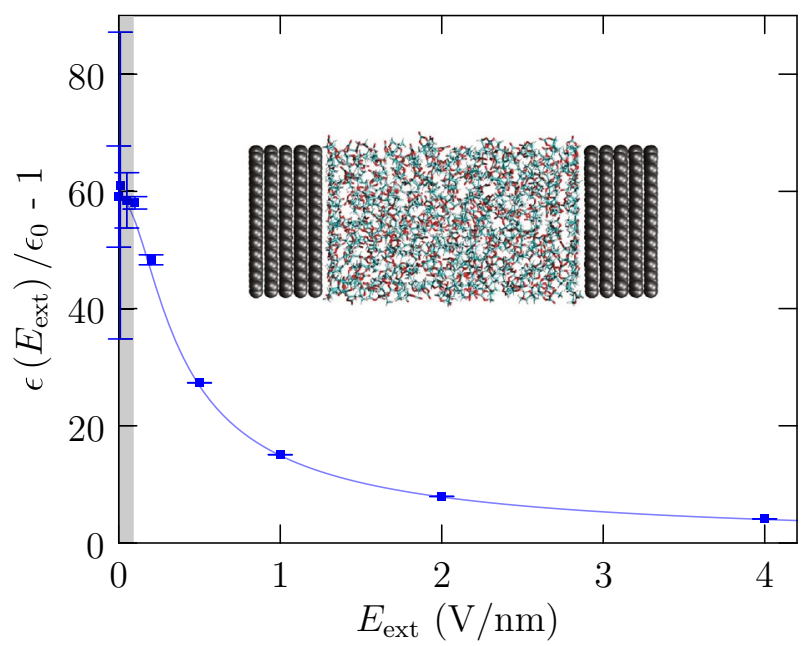

Fig. 8 For uniform liquid $\mathrm{PC}, \varepsilon\left(E_{\text {ext }}\right) / \varepsilon_{0}-1$ as a function of external electric field strength, redrawn from Yang et al. [82]. These molecular dynamics results were obtained for the uniform liquid with an applied uniform electric field. The error bars indicate a 95\% confidence interval. The curve is the fitted Booth model [62, 83-86]. The shaded region identifies the low-field regime based on the Booth model and that empirical parameterization. Evidently, the high-field behavior is simple in the model and the simulation. These calculations have been reexamined [87], and refined and extended [62]. Distinct from these uniform liquid calculations [82], the inset suggests how molecular-scale electric fields might be approached, i.e., by investigation of common lab-scale potential changes over nanometer-scale gaps

chemistry and the fact that the microstructures of CNT forests can be simple and controlled over interesting ranges [94]. Thus EDLCs based on CNT forests provide a comparatively simple and controllable setting to learn about the molecular solutions. Outstanding practical questions that call for better basic molecular-scale understanding include (a) the dependence of the capacitance on electrostatic potential [92, 94] and (b) the dependence of capacitance on pore sizes for mesoporous electrode materials $[95,96]$. Conclusive examination of those interesting questions will have to await further considerations. Here we make some primitive observations on work available so far.

One consideration for simulating these systems is the modeling of the electrodes. The simple model exemplified in Fig. 9 sets fixed charges based on appropriate preliminary calculations $[47,92]$. An alternative focuses on the conducting nature of the electrode and reformulates the simulations to incorporate a constraint of constant electric potential in a conducting phase [97-102]. The work of Wang et al. [97] studying $\mathrm{LiClO}_{4} /$ acetonitrile between planar graphite electrodes with a constant potential MD calculation provided a clarifying example. The distribution of the fluctuating charges on electrode atoms was simple (unimodal) for cases exploring a voltage window below $4 \mathrm{~V}$, though that situation changed markedly for net electric potential differences between the electrodes of $4 \mathrm{~V}$ and above. We note in passing that $4 \mathrm{~V}$ is close to the practical limit for the voltage window for experimental EDLC cases [94]. The complexities observed with the ultra-high potentials were associated with the depletion of the acetonitrile occupancy in $\mathrm{Li}^{+}$inner shells for $\mathrm{Li}^{+}$ions in close 

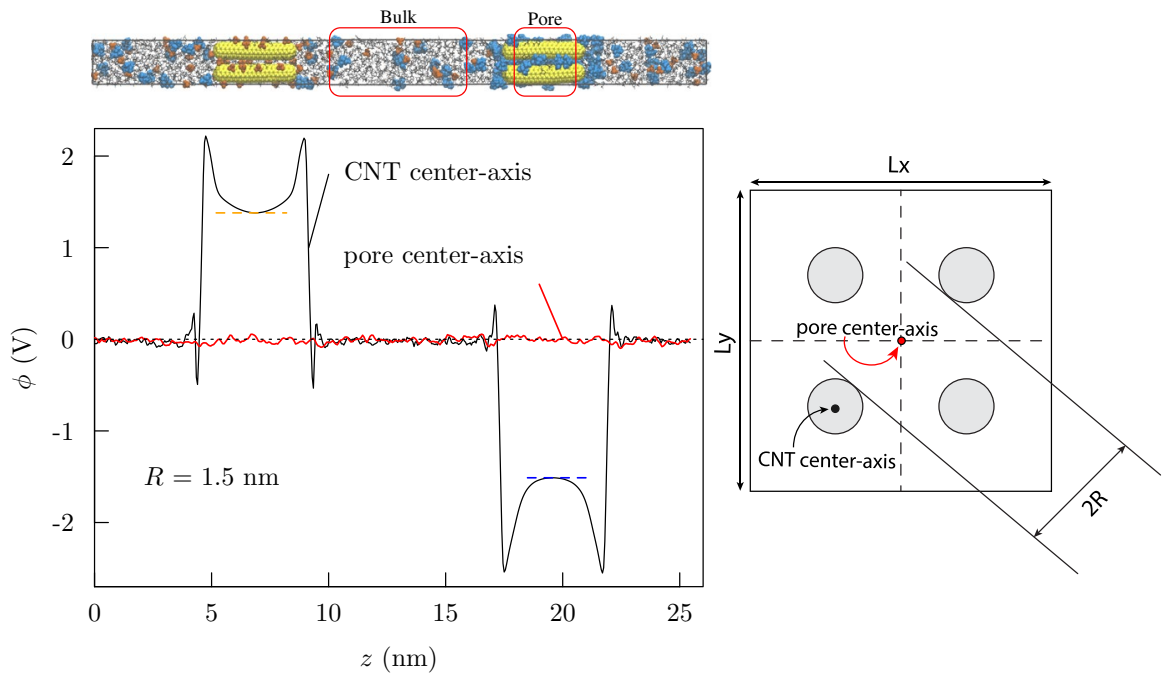

Fig. 9 Left top: a snapshot of a simulation box [47, 92] containing 4 positively (left) and 4 negatively (right) charged CNTs, each of $360^{\circ} \mathrm{C}$ atoms, with ions filling the pore regions around CNT. A total charge of $q= \pm N$ e is set on each nanotube with $N=(2,4,6,8,10)$ and a solution of $1 \mathrm{M} \mathrm{TEABF}_{4}$ (tetraethylammonium tetrafluoroborate) electrolyte (blue and orange, respectively) in propylene carbonate (sticks). The highest charged case, then, has $223 \mathrm{C} / \mathrm{gm}$, or adoption of $1300 \mathrm{~m}^{2} / \mathrm{gm}$ as a standard value for the specific area, $0.17 \mathrm{C} / \mathrm{m}^{2}$. The electrostatic potential was evaluated from the observed average charge density by numerical solution of the discretized Poisson equation [93]. Left bottom: a variation of electrostatic potential along the $z$-axis for a pore radius of $R=1.5 \mathrm{~nm}$ at a charge level of $N=6$. Right: a cross-section perpendicular to the $z$-axis defining the pore radius and the location of pore and CNT axis
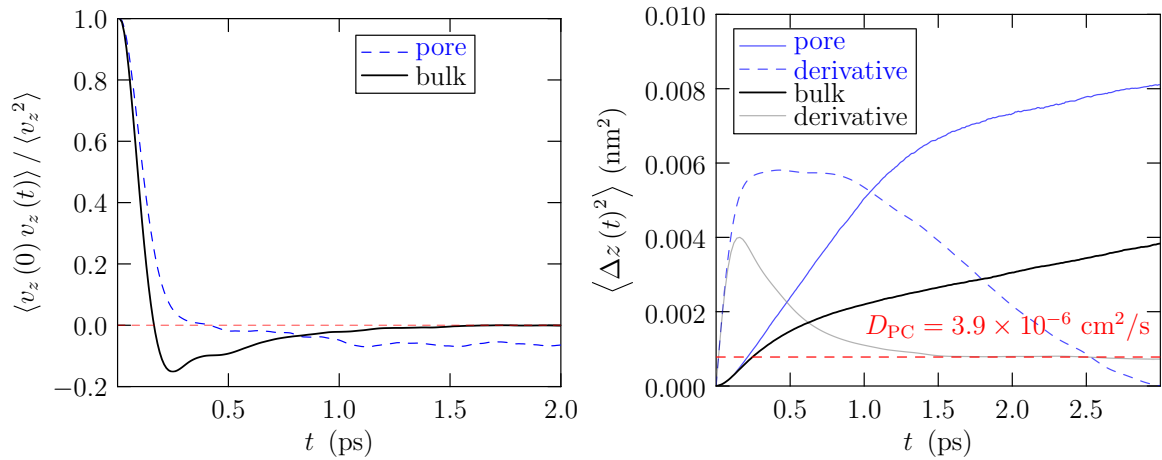

Fig. 10 Comparison of mobilities of PC molecules in bulk solution with PC molecules in the pore space of the CNT forest (Fig. 9) [47]. In this case, the CNTs are not charged and the pore radius (Fig. 9) is $R$ $=1 \mathrm{~nm}$. The indicated value of $D$ (right panel) is for PC molecules in the bulk solution. Notice that the mobility of PC molecules in the pore looks diffusive at intermediate times, but not for longer times here. Further, the suggested intermediate-time diffusive motion is faster in the pore, presumably an effect of preferential orientation of PC molecules in the pore space 
contact with the electrode. Those close contacts qualitatively change the density profiles of $\mathrm{Li}^{+}$ions with respect to the electrode, but do not qualitatively change the density profiles of the bigger $\mathrm{ClO}_{4}{ }^{-}$ion.

The physical conclusion is that the EDLC/CNT calculations discussed above with molecular ions (Fig. 9) conservatively aim for a range in which they provide a reasonable initial step, perhaps subject to subsequent refinement. We note additionally that applications of constant potential MD calculations, implemented in simulation packages such as LAMMPS [103], have been limited so far in the number of electrodes and their configurations, specifically to two-planar electrodes. We take up the important special case of $\mathrm{Li}^{+}$in the next section.

We emphasize that realistic molecular models of EDLC/CNTs are feasible for direct numerical simulation of the pore filling and the electrical characteristics $[47,91,92]$. This reduces possible uncertainty about solution composition in the pore spaces and permits study of the kinetics of the filling in realistic settings. That the relevant molecular mobilities are different in the pore spaces is already clear (Fig. 10).

\section{Empirically Scaled Partial Charges for $\mathrm{Li}^{+} . .$. Carbonate Interactions}

The results discussed in previous sections suggest that a parameterized, non-polarizable force field can reproduce experimental structural, thermodynamic, and dielectric properties of EC and PC liquids with acceptable accuracy [44, 46-48]. Next we consider the important case of addition of $\mathrm{Li}^{+}$ions to EC and PC. A primary concern is a valid description of the thermodynamics of $\mathrm{Li}^{+}$-solvent interactions. In view of the strength of those interactions, and non-linear behaviors exhibited in Fig. 8, these thermodynamic issues are not taken for granted. In setting revised models, we considered partial charges, empirically scaled on the basis of electronic structure calculations and available experimental results. The electronic structure-based methods employed [50] are (a) quasi-chemical theory for the thermodynamics, and (b) AIMD calculations for structural and mobility information.

We have compared results using partial charges available in the standard OPLSAA distribution for EC and PC solvents [104] to results derived from partial charges that were subsequently reduced to 90 and $80 \%$ of those values. Simulations treated a single $\mathrm{Li}^{+} \ldots \mathrm{PF}_{6}{ }^{-}$ion pair in both solvents. We compared structural and thermodynamics results with chemically based AIMD simulations.

\subsection{Free Energy Results and Quasi-Chemical Theory (QCT)}

QCT is based on the study of the occupancy of an inner shell of an $\mathrm{Li}^{+}$ion, here, by the carbonyl $\mathrm{O}$ atoms of the solvent. QCT provides the free energy, specifically the excess chemical potential, $\mu_{\mathrm{Li}^{+}}^{(\mathrm{ex})}$, for a solution phase $\mathrm{Li}^{+}$[50]. We use the cluster QCT method [105, 106]

$$
\mu_{\mathrm{Li}^{+}}^{(\mathrm{ex})}=-k T \ln K_{n}^{(0)} \rho_{\mathrm{sol}}{ }^{n}+\left(\mu_{\mathrm{Li}(\mathrm{sol})_{n}{ }^{+}}^{(\mathrm{ex})}-n \mu_{\mathrm{sol}}^{(\mathrm{ex})}\right),
$$


as a benchmark for comparison of QCT results for $\mu_{\mathrm{Li}^{+}}^{(\mathrm{ex})}$ obtained from MD simulation with simple force fields. In the first term, $K_{n}^{(0)}$ is the equilibrium ratio for the $\mathrm{Li}^{+}$ -solvent (sol) association reaction

$$
\mathrm{Li}^{+}+n \text { sol } \rightleftharpoons \mathrm{Li}(\mathrm{sol})_{n}^{+},
$$

treated as in an ideal gas phase; hence the superscript (0). The solvent density, $\rho_{\text {sol }}$, gauges the availability of solvent molecules to serve as ligands in this association, and this justifies the attention above to the equation of state of these liquids. The right-most term of Eq. (11) provides solvation of the $\mathrm{Li}(\mathrm{sol})_{n}{ }^{+}$complex by the solvation environment external to it. That, $\mu_{\mathrm{Li}\left(\mathrm{sol}_{n}{ }^{+}\right.}^{(\mathrm{e})}-n \mu_{\mathrm{sol}}^{(\mathrm{ex})}$, combination makes a favora-

ble contribution to the free energy.

For analyzing the MD results, we use the direct QCT approach [105]

$$
\mu_{\mathrm{Li}^{+}}^{(\mathrm{ex})} / R T=-\ln p^{(0)}\left(n_{\lambda}\right)+\ln \left\langle e^{\varepsilon / R T} \mid n_{\lambda}\right\rangle+\ln p\left(n_{\lambda}\right),
$$

which is tautologically related to Eq. (11) with the natural definition of the indicated probabilities [107]. $\varepsilon$ is the binding energy of the $\mathrm{Li}^{+}$. The advantage of this simulation-based QCT is that it permits calculation of solvation free energies, and correlation of those results with observed solution features.

The free energies of $\mathrm{Li}^{+}$transfer $\Delta \Delta G_{\mathrm{Li}^{+}}$to a carbonate solvent from water for the two QCT implementations (Fig. 11) compare accurate electronic structure calculations and classical FFMD simulation with simple force fields. The cluster QCT

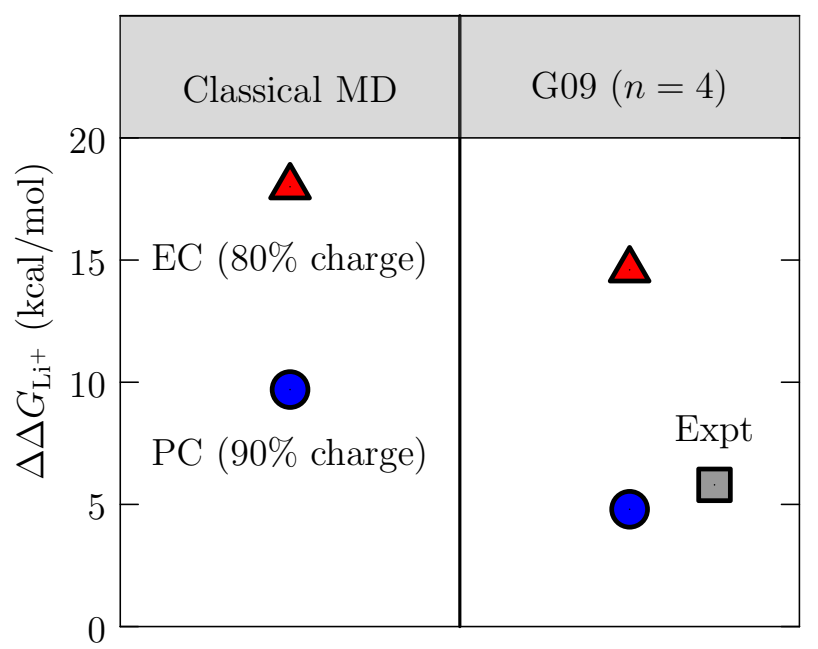

Fig. 11 Transfer free energies, $\Delta \Delta G_{\mathrm{Li}^{+}}$, comparing FFMD direct QCT results (left) and cluster-QCT results (right) using the G09 electronic structure software package. The cluster QCT results for the free energy of $\mathrm{Li}^{+}$transfer to PC from water agree with tabulated experimental values to within $1 \mathrm{kcal} / \mathrm{mol}$ [108]. Our experience using cluster QCT to predict ion hydration free energies [109-119] suggests that the G09 results have accuracy comparable to other ab initio predictions [120-123], and hence provide a useful benchmark for FFMD results 
result for the free energy of $\mathrm{Li}^{+}$transfer to $\mathrm{PC}$ from water agrees with the value tabulated by Marcus [108]. No experimental value is available for EC. The direct QCT evaluations of $\Delta \Delta G_{\mathrm{Li}^{+}}$for the FFMD simulations agree reasonably with the cluster QCT electronic structure calculations when the partial charges of the force fields are scaled by $80 \%$ (EC) or $90 \%$ (PC). The direct QCT MD calculations agree to within $2 \mathrm{kcal} / \mathrm{mol}$ on the $10-\mathrm{kcal} / \mathrm{mol}$ difference in transfer free energies between PC and EC predicted by the cluster QCT calculations.

Positive transfer free energies favor lithium ion solvation by water compared with either carbonate solvent. From the perspective of the cluster QCT calculations, the replacement free energy, reflecting the availability of the solvent molecules as ligands, is the foremost factor leading to that result. Comparing EC and PC transfer free energies, again from the perspective of cluster QCT, the solvation of the bare $\mathrm{EC} / \mathrm{PC}$ molecules serving as ligands is decisive in arriving at a positive free energy of transfer from PC to EC, with EC being slightly smaller.

\subsection{Radial Distribution Function}

Radial distributions (rdfs) of carbonyl $\mathrm{O}$ of $\mathrm{EC}$ and $\mathrm{PC}$ with $\mathrm{Li}^{+}$are sensitive to partial charges of an FFMD model (Fig. 12). AIMD results of $\mathrm{Li}^{+}$solvation in water [50] and PC agree with X-ray spectroscopy [34] and neutron diffraction [124, 125] results. Hence, we used AIMD for validation of FFMD results. The four-coordinate inner shell was observed in both AIMD and FFMD simulations of $\mathrm{Li}^{+}$solvation in EC and PC. The AIMD results match those from previous calculations [126]. The coordination number of $\mathrm{Li}^{+}$in PC using AIMD agrees with the 4.5 reported by neutron diffraction [125] and x-ray spectroscopy [34]. Interestingly, Bader charge analysis on AIMD configurations suggest that solvent molecules sometimes donate as much as 0.1 electron to an ion $[29,127]$. The neutron and X-ray diffraction experiments have the peak position at $2.04 \AA$, which is slightly longer than all FFMD results $(1.78-1.9 \AA)$, but comparable to AIMD $(2 \AA)$ and polarizable force field results (1.95-2 $\AA$ ). Yet, a four-coordinate inner solvation shell is seen consistently in all cases. The solvent density is less sensitive to these partial charges under the conditions of interest. Dielectric constants do change significantly with scaled partial charges, but the values realized are high enough that solvation characteristics are only slightly affected.

\subsection{Ion Mobilities}

The $\mathrm{Li}^{+}$msd results [128] were obtained from separate 1-ns simulations of one $\mathrm{Li}^{+}$ ....PF 6 ion pair in $249 \mathrm{EC}$ and $\mathrm{PC}$ molecules, and compared with the experimental values obtained from nuclear magnetic resonance (NMR) results [70]. The results (Fig. 14) for $90 \%$ charged PC and $80 \%$ charged EC were closest to the experimental results. The transference number, $t_{\mathrm{Li}^{+}}$, for $\mathrm{Li}^{+}$can be calculated from ratios of diffusion constants, $D$, according to

$$
t_{\mathrm{Li}^{+}}=\frac{D_{\mathrm{Li}^{+}}}{D_{\mathrm{Li}^{+}}+D_{\mathrm{PF}_{6}-}} .
$$




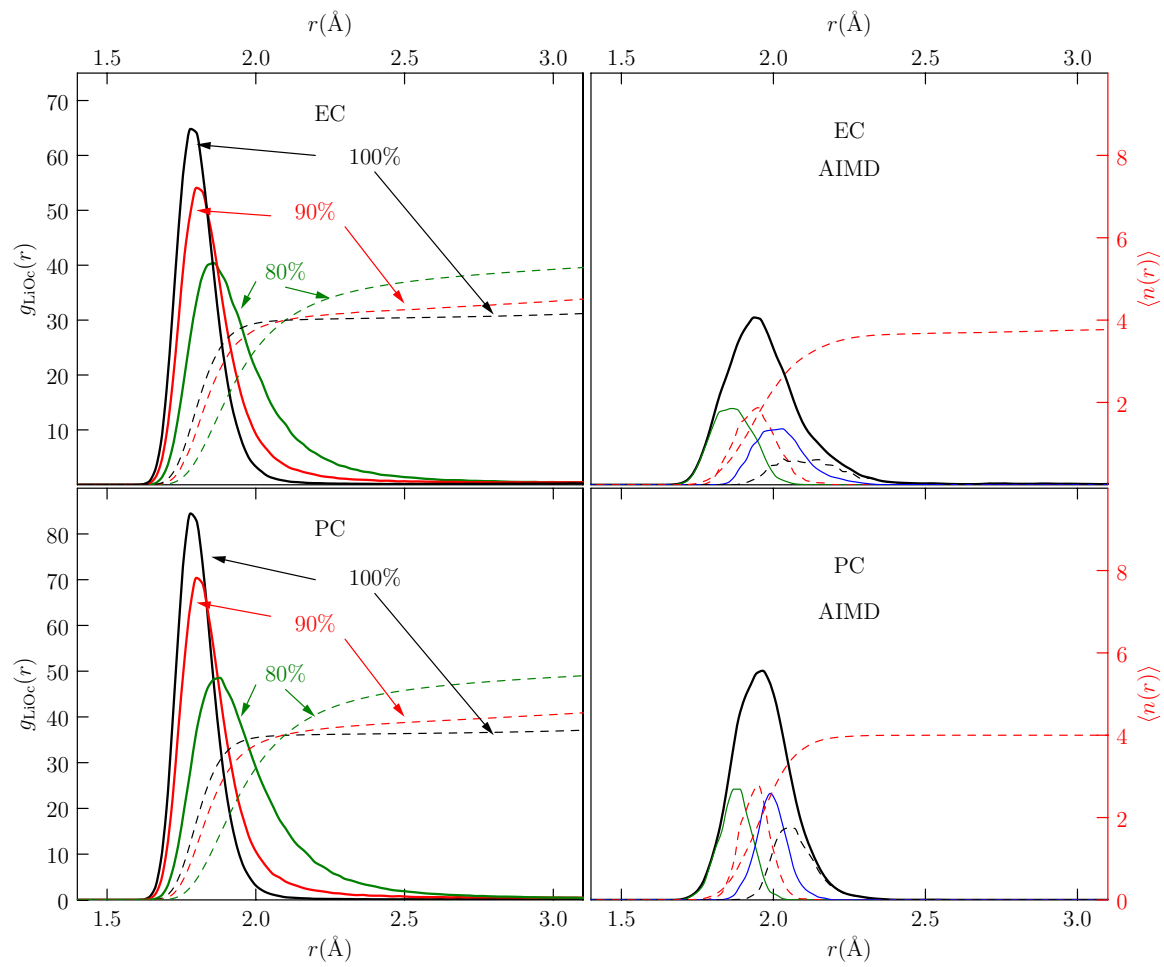

Fig. 12 Radial distributions of carbonyl $\mathrm{O}$ atoms from $\mathrm{Li}^{+}$in (top) EC and (bottom) PC using (left) FFMD and (right) AIMD simulations. Running coordination numbers (dashed curves and right axes) show near-neighbor (inner-shell) occupancies. In the FFMD cases, partial charges on EC and PC molecules were reduced from 100 to $90 \%$, and subsequently to $80 \%$. AIMD results show that four solvent molecules fully saturate the $\mathrm{Li}^{+}$coordination. The FFMD results demonstrate the importance of repulsions between near-neighbor (inner-shell) solvating molecules: the occupancy of the inner shell increases moderately, and inner-shell structures broaden, as the solvent partial charges are scaled down

The computed transference numbers of 0.35 (EC) and 0.31 (PC) are consistent with previous NMR [70] and impedance spectroscopy (EIS) [128] experiments. The diffusion constant value changes significantly with partial charges of solvent but the transference numbers are less sensitive.

In summary, changes to the partial charges on PC and EC solvents alter solvation structure and transport properties of $\mathrm{Li}^{+}$and $\mathrm{PF}_{6}^{-}$ions. Based on our results for radial distribution functions (Fig. 12), diffusion constants (Fig. 13), and transference numbers (Fig. 14), we identify $90 \%$ scaling of PC partial charges and $80 \%$ scaling for EC. 


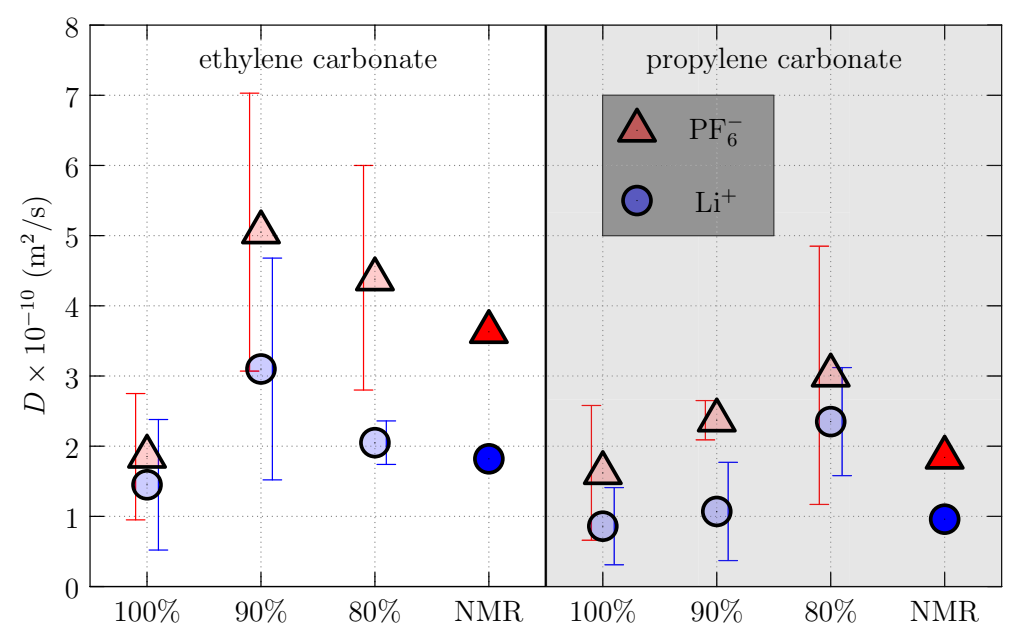

Fig. 13 Diffusion constants were calculated from FFMD using the mean-squared displacement (msd) of $\mathrm{Li}^{+}$and $\mathrm{PF}_{6}{ }^{-}$ions in EC (left) and PC (right). The msd was calculated for $25 \mathrm{ps}$. Experimental values are taken from Hayamizu et al. [70] As evident from these plots, the diffusion constants match experimental values best for $80 \%$ of partial charges on EC and $90 \%$ on PC

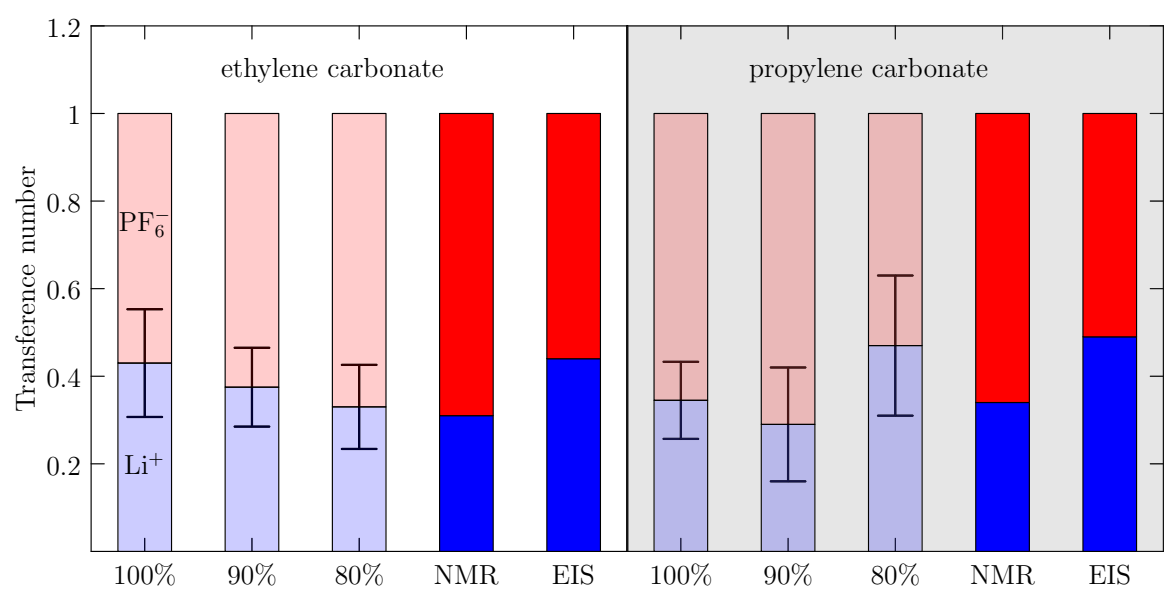

Fig. 14 Transference numbers for $\mathrm{Li}^{+}$and $\mathrm{PF}_{6}^{-}$in $\mathrm{EC}$ (left) and $\mathrm{PC}$ (right) were calculated using FFMD and Eq. 14. The NMR diffusion constants at $1 \mathrm{M}$ salt concentration were used to calculate experimental transference numbers (NMR) [70] and the combined AC impedance and DC polarization results apply to $0.1 \mathrm{M}$ salt concentration (EIS). The experimental results show little dependence on salt concentration, and the calculated results agree with the experimental numbers

\section{Model solid electrolyte interphase layer}

The discussion above encourages us to apply such an empirical non-polarizable force field to study non-aqueous electrolytes more broadly. Thus we extended our 

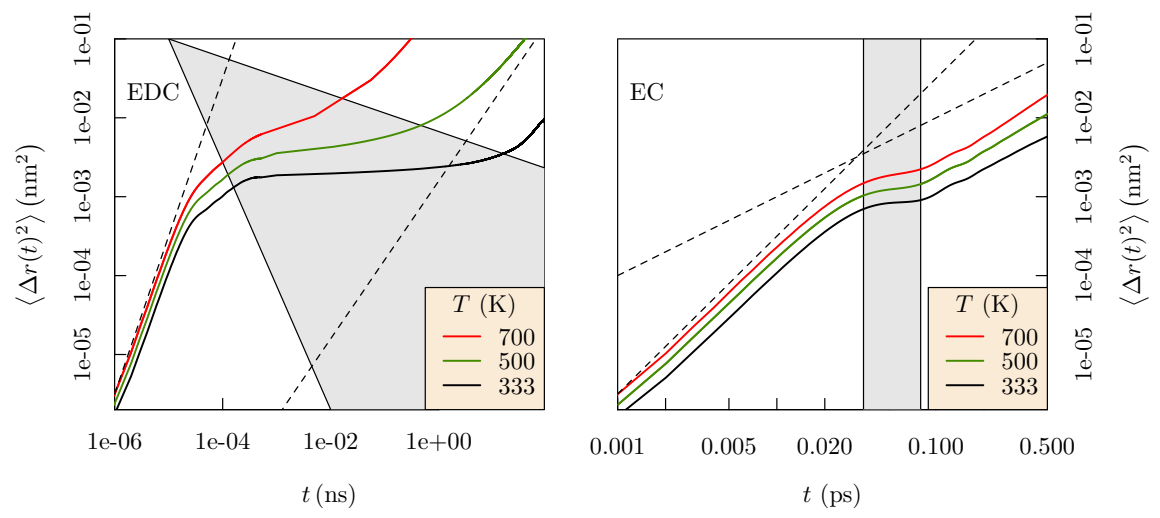

Fig. 15 Mean-squared displacements for $\mathrm{Li}^{+}$in EDC and EC. The behavior in EDC at intermediate timescales $0.001 \mathrm{~ns}<t<1 \mathrm{~ns}$ demonstrates trapping of the $\mathrm{Li}^{+}$ion (gray shaded area). Ballistic motion is evident at short timescales, and diffusive motion at long timescales in both EDC and EC solvents. At high $T$, the trapping regime shrinks. Note the time scales differ dramatically between the left and right panels here. In the model SEI (left panel), trapping times are longer than nanoseconds. Thus for direct simulation of trapping, simulations should access multiples of that nanosecond time scale

effort to simulate $\mathrm{Li}^{+}$ion transport within a model SEI layer of dilithium ethylene dicarbonate (EDC) [129]. The $\mathrm{Li}^{+}$ion $\mathrm{msd}$ in EDC and EC solvents (Fig. 15) shows three distinct temporal regions corresponding to ballistic, trapping, and diffusive motions. The trapping region for $\mathrm{Li}^{+}$ion is extended for glassy EDC material and has significant temperature dependence compared to liquid EC solvent. Further analysis [129] confirmed the glassy behavior of the EDC matrix.

\section{Conclusions}

The basic results discussed suggest that an empirically parameterized, non-polarizable force field can reproduce experimental structural, thermodynamic, and dielectric properties of EC and PC liquids with acceptable accuracy. More sophisticated force fields might include molecular polarizability and Buckingham-model description of inter-atomic overlap repulsions as extensions of Lennard-Jones models of van der Waals interactions. Simple approaches should be similarly successful also for applications to organic molecular ions in EC/PC solutions, but the important case of $\mathrm{Li}^{+}$deserves special attention because of the particularly strong interactions of that small ion with neighboring solvent molecules. To treat the $\mathrm{Li}^{+}$ions in liquid EC/PC solutions, we identify interaction models defined by empirically scaled partial charges for ion-solvent interactions. The empirical adjustments use more basic inputs, electronic structure calculations and AIMD simulations, and also experimental results on $\mathrm{Li}^{+}$thermodynamics and transport in EC/PC solutions. Application of such models to the mechanism of $\mathrm{Li}^{+}$transport in glassy SEI models emphasizes the 
advantage of long time-scale molecular dynamics studies of these non-equilibrium materials.

Acknowledgements Sandia National Laboratories (SNL) is a multi-mission laboratory managed and operated by National Technology and Engineering Solutions of Sandia, LLC., a wholly owned subsidiary of Honeywell International, Inc., for the US Department of Energy's National Nuclear Security Administration under contract DE-NA-0003525. This work is supported by the Assistant Secretary for Energy Efficiency and Renewable Energy, Office of Vehicle Technologies of the US Department of Energy under contract no. DE-AC02-05CH11231, subcontract no. 7060634 under the Advanced Batteries Materials Research (BMR) Program and Sandia's LDRD program (MIC and SBR). This work was performed, in part, at the Center for Integrated Nanotechnologies (CINT), an Office of Science User Facility operated for the U.S. DOE's Office of Science by Los Alamos National Laboratory (contract DE-AC5206NA25296) and SNL.

Open Access This article is distributed under the terms of the Creative Commons Attribution 4.0 International License (http://creativecommons.org/licenses/by/4.0/), which permits unrestricted use, distribution, and reproduction in any medium, provided you give appropriate credit to the original author(s) and the source, provide a link to the Creative Commons license, and indicate if changes were made.

\section{References}

1. Schütter C, Husch T, Korth M, Balducci A (2015) Toward new solvents for EDLCs: from computational screening to electrochemical validation. J Phys Chem C 119:13413-13424

2. Xu K (2014) Electrolytes and interphases in Li-Ion batteries and beyond. Chem Rev 114:11503-11618

3. Berendsen HJC, Postma JPM, van Gunsteren WF, Hermans J (1981) In: Intermolecular Forces: Proceedings of the Fourteenth Jerusalem Symposium on Quantum Chemistry and Biochemistry Held in Jerusalem, Israel, April 13-16, 1981; Pullman, B., Ed.; Springer Netherlands: Dordrecht

4. Postma JPM (1985) MD of H2O. A molecular dynamics study of water. Ph.D. thesis, 1985; University of Groningen

5. Berendsen HJC, Grigera JR, Straatsma TP (1987) The missing term in effective pair potentials. J Phys Chem 91:6269-6271

6. Pohorille A, Pratt LR (2012) Is water the universal solvent for life? Orig Life Evol Biosph 42:405-409

7. Borodin O (2009) Polarizable force field development and molecular dynamics simulations of ionic liquids. J Phys Chem B 113:11463-11478

8. Korth M (2014) In: Chem Modell; Royal Society of Chemistry: Cambridge, 2014; pp 57-87

9. Husch T, Korth M (2015) How to estimate solid-electrolyte-interphase features when screening electrolyte materials. Phys Chem Chem Phys 17:1-10

10. Zhong C, Hu W (2016) Electrolytes for electrochemical supercapacitors. CRC Press, Boca Raton, pp 31-254

11. Korth M (2014) Large-scale virtual high-throughput screening for the identification of new battery electrolyte solvents: evaluation of electronic structure theory methods. Phys Chem Chem Phys 16:7919-7926

12. Husch T, Yilmazer ND, Balducci A, Korth M (2014) Large-scale virtual high-throughput screening for the identification of new battery electrolyte solvents: computing infrastructure and collective properties. Phys Chem Chem Phys 17:1-8

13. Husch T, Korth M (2015) Charting the known chemical space for non-aqueous lithium-air battery electrolyte solvents. Phys Chem Chem Phys 17:22596-22603

14. Conway BE (2013) Electrochemical supercapacitors: scientific fundamentals and technological applications. Springer Science \& Business Media, New York

15. Yang L, Fishbine BH, Migliori A, Pratt LR (2009) Molecular simulation of electric double-layer capacitors based on carbon nanotube forests. J Am Chem Soc 131(34):12373-12376

16. Tanaike O, Tanai, Futaba DN, Hata K, Hatori H (2009) Supercapacitors using pure single-walled carbon nanotubes. Carbon Lett 10:90-93 
17. Hata K, Futaba DN, Mizuno K, Namai T, Yumura M, Iijima S (2004) Water-assisted highly efficient synthesis of impurity-free single-walled carbon nanotubes. Science 306:1362-1364

18. Baughman RH, Zakhidov AA, de Heer WA (2004) Carbon nanotubes-the route toward applications. Science 297:787-792

19. Oguntoye M, Oak S, Pashazanusi L, Pratt L, Pesika NS (2017) Vertically-aligned carbon nanotube arrays as binder-free supports for nickel cobaltite based faradaic supercapacitor electrodes. Electrochim Acta 236:408-416

20. Soto FA, Ma Y, Martinez de la Hoz JM, Seminario JM, Balbuena PB (2015) Formation and growth mechanisms of solid-electrolyte interphase layers in rechargeable batteries. Chem Mat 27:7990-8000

21. An SJ, Li J, Daniel C, Mohanty D, Nagpure S, Wood DL III (2016) The state of understanding of the lithium-ion-battery graphite solid electrolyte interphase (SEI) and its relationship to formation cycling. Carbon 105:52-76

22. Leung K, Budzien JL (2010) Ab initio molecular dynamics simulations of the initial stages of solid electrolyte interphase formation on lithium ion battery graphitic anodes. Phys Chem Chem Phys 12:6583-6586

23. Zhuang G, Xu K, Yang H, Jow T, Ross PJ (2005) Lithium ethylene dicarbonate identified as the primary product of chemical and electrochemical reduction of EC in 1.2 M LiPF6/EC:EMC electrolyte. J Phys Chem B 109:17567-73

24. Martinez de la Hoz JM, Soto FA, Balbuena PB (2015) Effect of the electrolyte composition on SEI reactions at Si anodes of Li-Ion batteries. J Phys Chem C 119:7060-68

25. Borodin O, Zhuang GV, Ross PN, Xu K (2013) Molecular dynamics simulations and experimental study of lithium ion transport in dilithium ethylene dicarbonate. J Phys Chem C 117:7433-7444

26. Borodin O, Bedrov D (2014) Interfacial structure and dynamics of the lithium alkyl dicarbonate SEI components in contact with the lithium battery electrolyte. J Phys Chem C 118:18362-18371

27. Benitez L, Cristancho D, Seminario JM, Martinez de la Hoz JM, Balbuena PB (2014) Electron transfer through solid-electrolyte-interphase layers formed on $\mathrm{Si}$ anodes of Li-ion batteries. Electrochim Acta 140:250-257

28. Ong MT, Verners O, Draeger EW, van Duin ACT, Lordi V, Pask JE (2015) Lithium ion solvation and diffusion in bulk organic electrolytes from first-principles and classical reactive molecular dynamics. J Phys Chem B 119:1535-1545

29. Zhang W, Pratt LR (2015) AIMD results for a concentrated solution of tetra-ethylammonium tetrafluoroborate in propylene carbonate. ECS Trans 66:1-5

30. Borodin O, Olguin M, Ganesh P, Kent PRC, Allen JL, Henderson WA (2016) Competitive lithium solvation of linear and cyclic carbonates from quantum chemistry. Phys Chem Chem Phys 18:164-175

31. Kumar N, Seminario JM (2016) Lithium-ion model behavior in an ethylene carbonate electrolyte using molecular dynamics. J Phys Chem C 120:16322-16332

32. Arslanargin A, Powers A, Beck TL, Rick SW (2016) Models of ion solvation thermodynamics in ethylene carbonate and propylene carbonate. J Phys Chem B 120:1497-1508

33. Pollard TP, Beck TL (2017) Structure and polarization near the Li+ ion in ethylene and propylene carbonates. J Chem Phys 147:161710

34. Smith JW, Lam RK, Sheardy AT, Shih O, Rizzuto AM, Borodin O, Harris SJ, Prendergast D, Saykally RJ (2014) X-ray absorption spectroscopy of $\mathrm{LiBF}_{4}$ in propylene carbonate: a model lithium ion battery electrolyte. Phys Chem Chem Phys 16:23568-23575

35. Van Der Spoel D, Lindahl E, Hess B, Groenhof G, Mark AE, Berendsen HJC (2005) GROMACS: fast, flexible, and free. J Comput Chem 26:1701-1718

36. Jorgensen WL, Maxwell DS (1996) Development and testing of the OPLS all-atom force field on conformational energetics and properties of organic liquids. J Am Chem Soc 118:11225-11236

37. Delavoux YM, Gilmore M, Atkins MP, Swad ba Kwa ny MG, Holbrey JD (2017) Intermolecular structure and hydrogen-bonding in liquid 1,2-propylene carbonate and 1,2-glycerol carbonate determined by neutron scattering. Phys Chem Chem Phys 19:2867-2876

38. Parrinello M, Rahman A (1981) Polymorphic transitions in single crystals: a new molecular dynamics method. J Appl Phys 52:7182-7190

39. Nosé S (1984) A molecular dynamics method for simulations in the canonical ensemble. Mol Phys 52:255-268

40. Hoover WG (1985) Canonical dynamics: equilibrium phase-space distributions. Phys Rev A $31: 1695-1697$ 
41. Hess B, Bekker H, Berendsen H, Fraaije GEM (1997) LINCS: a linear constraint solver for molecular simulations. J Comp Chem 18:1463-1472

42. Bedrov D, Borodin O, Hooper JB (2017) Li+ transport and mechanical properties of model solid electrolyte interphases (SEI): insight from atomistic molecular dynamics simulations. J Phys Chem C 121(30):16098-16109

43. Zhu P, You X, Pratt L, Papadopoulos K (2011) Generalizations of the Fuoss approximation for ion pairing. J Chem Phys 134:054502

44. You X, Chaudhari MI, Pratt LR, Pesika N (2013) Interfaces of propylene carbonate. J Chem Phys 138:114708

45. Zhu P, Pratt L, Papadopoulos K (2012) Pairing of 1-hexyl-3-methylimidazolium and tetrafluoroborate ions in n-pentanol. J Chem Phys 137:174501

46. You X, Pratt LR, Rick, SW (2014) The role of attractive interactions in the dynamics of molecules in liquids. arXiv:1411.1773

47. You X (2014) Interfacial characteristics of propylene carbonate and validation of simulation models for electrochemical applications. PhD thesis, Department of Chemical and Biomolecular Engineering, Tulane University

48. You X, Chaudhari MI, Rempe SB, Pratt LR (2016) Dielectric relaxation of ethylene carbonate and propylene carbonate from molecular dynamics simulations. J Phys Chem B 120:1849-1853

49. Schäffner B, Schäffner F, Verevkin SP, Börner A (2010) Organic carbonates as solvents in synthesis and catalysis. Chem Rev 110:4554-4581

50. Chaudhari MI, Nair JR, Pratt LR, Soto FA, Balbuena PB, Rempe SB (2016) Scaling atomic partial charges of carbonate solvents for lithium ion solvation and diffusion. J Chem Theory Comp 12:5709-5718

51. Liu S, Hu Z, Weeks JD, Fourkas JT (2012) Structure of liquid propionitrile at interfaces. 1. Molecular dynamics simulations. J Phys Chem C 116:4012-4018

52. Ding F, Rivera CA, Zhong Q, Manfred K, He X, Brindza MR, Walker RA, Fourkas JT (2012) Structure and dynamics of trimethylacetonitrile at the silica/vapor, silica/liquid, and liquid/vapor interfaces. J Phys Chem C 116:7000-7009

53. Berne BJ, Fourkas JT, Walker RA, Weeks JD (2016) Nitriles at silica interfaces resemble supported lipid bilayers. Accts Chem Res 49:1605-1613

54. Hsu C, Chandler D (1978) RISM calculation of the structure of liquid acetonitrile. Mol Phys 36:215-224

55. Böhm H, McDonald I, Madden P (1983) An effective pair potential for liquid acetonitrile. Mol Phys 49:347-360

56. Jorgensen WL, Briggs JM (1988) Monte Carlo simulations of liquid acetonitrile with a three-site model. Mol Phys 63:547-558

57. Hu Z, Weeks JD (2010) Acetonitrile on silica surfaces and at its liquid? Vapor interface: structural correlations and collective dynamics. J Phys Chem C 114:10202-10211

58. Stoppa A, Nazet A, Buchner R, Thoman A, Walther M (2015) Dielectric response and collective dynamics of acetonitrile. J Mol Liq 212:963-968

59. Ding F, Hu Z, Zhong Q, Manfred K, Gattass RR, Brindza MR, Fourkas JT, Walker RA, Weeks JD (2010) Interfacial organization of acetonitrile: simulation and experiment. J Phys Chem C 114:17651-17659

60. Cheng L, Morrone JA, Berne BJ (2012) Structure and dynamics of acetonitrile confined in a silica nanopore. J Phys Chem C 116:9582-9593

61. Pothoczki S, Pusztai L (2017) Intermolecular orientations in liquid acetonitrile: new insights based on diffraction measurements and all-atom simulations. J Mol Liq 225:160-166

62. Daniels IN, Wang Z, Laird BB (2017) Dielectric properties of organic solvents in an electric field. J Phys Chem C 121:1025-1031

63. Li Y, Leung K, Qi Y (2016) Computational exploration of the Li-electrodelelectrolyte interface in the presence of a nanometer thick solid-electrolyte interphase layer. Accts Chem Res 49:2363-2370

64. Tyunina EY, Chekunova MD (2017) Physicochemical properties of binary solutions of propylene carbonate-acetonitrile in the range of 253.15-313.15 K. Russ J Phys Chem A 91:894-900

65. You X, Chaudhari MI, Pratt LR, Pesika N, Aritakula KM, Rick SW (2015) Erratum: Interfaces of propylene carbonate [J. Chem. Phys. 138, 114708 (2013)]. J Chem Phys 142:249902

66. Wilson GM, Von Niederhausern DM, Giles NF (2002) Critical point and vapor pressure measurements for nine compounds by a low residence time flow method. J Chem Eng Data 47:761-764

67. Zwanzig R (2001) Nonequilibrium statistical mechanics. Oxford, London 
68. Zhu P, Pratt LR, Papadopoulos KD (2012) Pairing of 1-hexyl-3-methylimidazolium and tetrafluoroborate ions in n-pentanol. J Chem Phys 137:174501

69. Wolynes PG (1978) Molecular theory of solvated ion dynamics. J Chem Phys 68:473

70. Hayamizu K (2012) Temperature dependence of self-diffusion coefficients of ions and solvents in ethylene carbonate, propylene carbonate, and diethyl carbonate single solutions and ethylene Carbonate + Diethyl Carbonate Binary Solutions of LiPF $_{6}$ Studied by NMR. J Chem Eng Data 57:2012-2017

71. Payne R, Theodorou IE (1972) Dielectric properties and relaxation in ethylene carbonate and propylene carbonate. J Phys Chem 76:2892-2900

72. Tasaki K, Harris SJ (2010) Computational study on the solubility of lithium salts formed on lithium ion battery negative electrode in organic solvents. J Phys Chem C 114:8076-8083

73. McQuarrie D (2000) Statistical mechanics. University Science Books, Davis, California

74. Smyth CP (1955) Dielectric behavior and structure; dielectric constant and loss dipole moment and molecular structure. McGraw-Hill, New York

75. Williams G (1979) Molecular aspects of multiple dielectric relaxation processes in solid polymers. Electr Phen Polym Sci 33:59-92

76. Borodin O, Bedrov D, Smith GD (2002) Molecular dynamics simulation study of dielectric relaxation in aqueous poly(ethylene oxide) solutions. Macromolecules 35:2410-2412

77. Nandi N, Bhattacharyya K, Bagchi B (2000) Dielectric relaxation and solvation dynamics of water in complex chemical and biological systems. Chem Rev 100:2013-2046

78. Williams G, Watts DC (1970) Non-symmetrical dielectric relaxation behaviour arising from a simple empirical decay function. Trans Faraday Soc 66:80-85

79. Hosamani MT, Ayachit NH, Deshpande DK (2008) The dielectric studies on some substituted esters. J Mol Liq 137:43-45

80. Zhang L, Greenfield ML (2007) Relaxation time, diffusion, and viscosity analysis of model asphalt systems using Mol. Sim J Chem Phys 127:194502

81. Eisenberg DS, Kauzmann W (1969) The structure and properties of water. Clarendon Press, Oxford, London, Vol. 123; See Table 4.5, p. 207

82. Yang L, Fishbine BH, Migliori A, Pratt LR (2010) Dielectric saturation of liquid propylene carbonate in electrical energy storage applications. J Chem Phys 132:044701

83. Booth F (1951) The dielectric constant of water and the saturation effect. J Chem Phys 19:391-394

84. Yeh I-C, Berkowitz ML (1999) Dielectric constant of water at high electric fields: molecular dynamics study. J Chem Phys 110:7935

85. Dzubiella J, Hansen J-P (2005) Electric-field-controlled water and ion permeation of a hydrophobic nanopore. J Chem Phys 122:234706

86. Apol MEF, Amadei A, Di Nola A (2002) Statistical mechanics and thermodynamics of magnetic and dielectric systems based on magnetization. J Chem Phys 116:4426-4436

87. Wang H, Varghese J, Pilon L (2011) Simulation of electric double layer capacitors with mesoporous electrodes: effects of morphology and electrolyte permittivity. Electrochim Acta 56:6189-6197

88. Matyushov DV (2015) Nonlinear dielectric response of polar liquids. J Chem Phys 142:244502-6

89. Fulton RL (2016) Comment on "Nonlinear dielectric response of polar liquids" [J. Chem. Phys. 142, 244502 (2015)]. J Chem Phys 144:087101-3

90. Matyushov DV (2016) Response to comment on nonlinear dielectric response of polar liquids [J. Chem. Phys. 144, 087101 (2016)]. J Chem Phys 144:087102

91. Yang L, Fishbine BH, Migliori A, Pratt LR (2010) Dielectric saturation of liquid propylene carbonate in electrical energy storage applications. J Chem Phys 132:044701

92. Muralidharan A, You X, Pratt L, Hoffman G (2017) Supercapacitors based on carbon-nanotube forests. APS March Meet 62(4)

93. Muralidharan A, Fujioka H (2017) Poisson solver: GITHUB repository. https://doi.org/10.5281/ zenodo. 580088

94. Tanaike O, Imoto K, Futaba D, Hata K, Hatori H (2009) Supercapacitors using pure single-walled carbon nanotubes. Carbon Lett 10:90-93

95. Chmiola J, Yushin G, Gogotsi Y, Portet C, Simon P, Taberna PL (2006) Anomalous increase in carbon capacitance at pore sizes less than 1 nanometer. Science 313(5794):1760-1763. https://doi. org/10.1126/science. 1132195

96. Centeno TA, Sereda O, Stoeckli F (2011) Capacitance in carbon pores of 0.7 to $15 \mathrm{~nm}$ : a regular pattern. Phys Chem Chem Phys 13:12403-12406 
97. Wang Z, Yang Y, Olmsted DL, Asta M, Laird BB (2014) Evaluation of the constant potential method in simulating electric double-layer capacitors. J Chem Phys 141:184102

98. Reed SK, Lanning OJ, Madden PA (2007) Electrochemical interface between an ionic liquid and a model metallic electrode. J Chem Phys 126:084704

99. Siepmann JI, Sprik M (1995) Influence of surface topology and electrostatic potential on water/ electrode systems. J Chem Phys 102:511-524

100. Vatamanu J, Bedrov D, Borodin O (2017) On the application of constant electrode potential simulation techniques in atomistic modelling of electric double layers. Mol Sim 43:838-849

101. Matsumi Y, Nakano H, Sato H (2017) Constant-potential molecular dynamics simulations on an electrode-electrolyte system: calculation of static quantities and comparison of two polarizable metal electrode models. Chem Phys Lett 681:80-85

102. Petersen MK, Kumar R, White HS, Voth GA (2012) A computationally efficient treatment of polarizable electrochemical cells held at a constant potential. J Phys Chem C 116:4903-4912

103. Plimpton S (1995) Fast parallel algorithms for short-range molecular dynamics. J Comp Phys $117: 1-19$

104. Soetens J-C, Millot C, Maigret B, Bakó I (2001) Molecular dynamics simulation and X-ray diffraction studies of ethylene carbonate, propylene carbonate and dimethyl carbonate in liquid phase. $\mathrm{J}$ Mol Liq 92:201-216

105. Rogers DM, Jiao D, Pratt LR, Rempe SB (2012) In: Annual Reports in Computational Chemistry. Wheeler R (Ed.) Elsevier; pp 71-127

106. Sabo D, Varma S, Martin MG, Rempe SB (2008) Studies of the thermodynamic properties of hydrogen gas in bulk water. J Phys Chem B 112:867-876

107. Asthagiri D, Dixit PD, Merchant S, Paulaitis ME, Pratt LR, Rempe SB, Varma S (2010) Ion selectivity from local configurations of ligands in solutions and ion channels. Chem Phys Lett 485:1-7

108. Marcus Y (1983) Thermodynamic functions of transfer of single ions from water to nonaqueous and mixed solvents: part 1- Gibbs free energies of transfer to nonaqueous solvents. Pure Appl Chem 55:977-1021

109. Pratt LR, Rempe SB (1999) In: Simulation and theory of electrostatic interactions in solution. Hummer G, Pratt LR (Eds.) AIP Conf. Proc., AIP Press, New York, NY; Vol. 492; pp 172-201

110. Rempe SB, Pratt LR, Hummer G, Kress JD, Martin RL, Redondo A (2000) The hydration number of $\mathrm{Li}^{+}$in liquid water. J Am Chem Soc 122:966-967

111. Rempe SB, Pratt LR (2001) The hydration number of $\mathrm{Na}^{+}$in liquid water. Fl Phase Eq 183:121-132

112. Rempe SB, Asthagiri D, Pratt LR (2004) Inner shell definition and absolute hydration free energy of $\mathrm{K}^{+}(\mathrm{aq})$ on the basis of quasi-chemical theory and $\mathrm{Ab}$ initio molecular dynamics. Phys Chem Chem Phys 6:1966-1969

113. Asthagiri D, Pratt LR, Paulaitis ME, Rempe SB (2004) Hydration structure and free energy of biomolecularly specific aqueous dications, including $\mathrm{Zn}^{2+}$ and first transition row metals. J Am Chem Soc 126:1285-1289

114. Varma S, Rempe SB (2008) Structural transitions in ion coordination driven by changes in competition for ligand binding. J Am Chem Soc 130:15405-15419

115. Jiao D, Leung K, Rempe SB, Nenoff TM (2011) First principles calculations of atomic nickel redox potentials and dimerization free energies: a study of metal nanoparticle growth. J Chem Theo Comp 7:485-495

116. Sabo D, Jiao D, Varma S, Pratt LR, Rempe SB (2013) Case Study of $\mathrm{Rb}^{+}(\mathrm{aq})$, quasi-chemical theory of ion hydration, and the no split occupancies rule. Ann Rep Sect C (Phys. Chem.) 109:266-278

117. Chaudhari MI, Soniat M, Rempe SB (2015) Octa-coordination and the aqueous $\mathrm{Ba}^{2+}$ ion. J Phys Chem B 119:8746-8753

118. Stevens MJ, Rempe SLB (2016) Ion-specific effects in carboxylate binding sites. J. Phys. Chem. B 120:12519-12530

119. Chaudhari MI, Pratt LR, Rempe SB (2018) Utility of chemical computations in predicting solution free energies of metal ions. Mol Simul 44(2):110-116

120. Yanase S, Oi T (2002) Solvation of lithium ion in organic electrolyte solutions and its isotopie reduced partition function ratios studied by $\mathrm{Ab}$ initio molecular orbital method. J Nucl Sci Tech 39:1060-1064

121. Åqvist J (1990) Ion-water interaction potentials derived from free energy perturbation simulations. J Phys Chem 94:8021-8024 
122. Leung K, Rempe SB, von Lilienfeld OA (2009) Ab initio molecular dynamics calculations of ion hydration free energies. J Chem Phys 130:204507-204517

123. Bhatt MD, Cho M, Cho K (2010) Interaction of $\mathrm{Li}^{+}$Ions with ethylene carbonate (EC): density functional theory calculations. Appl Surf Sci 257:1463-1468

124. Mason PE, Ansell S, Neilson GW, Rempe SB (2015) Neutron scattering studies of the hydration structure of $\mathrm{Li}^{+}$. J Phys Chem B 119:2003-2009

125. Kameda Y, Umebayashi Y, Takeuchi M, Wahab MA, Fukuda S, Ishiguro S-I, Sasaki M, Amo Y, Usuki T (2007) Solvation structure of $\mathrm{Li}^{+}$in concentrated $\mathrm{LiPF}_{6}$-propylene carbonate solutions. J Phys Chem B 111:6104-6109

126. Ganesh P, Jiang D, Kent PRC (2011) Accurate static and dynamic properties of liquid electrolytes for Li-Ion batteries from Ab initio molecular dynamics. J Phys Chem B 115:3085-3090

127. Tang W, Sanville E, Henkelman G (2009) A grid-based Bader analysis algorithm without lattice bias. J Phys Condens Matter 21:084204

128. Muralidharan A., Pratt LR, Chaudhari MI, Rempe SB (2018) Comparison of single-ion molecular dynamics in common solvents. arXiv:1801.07116

129. Muralidharan A, Chaudhari M, Rempe SB, Pratt LR (2017) Molecular dynamics simulations of lithium ion transport through solid electrolyte interface layer. ECS Trans 77(11):1155-1162 